Pathophysiology

of Haemostasis and Thrombosis
Pathophysiol Haemost Thromb 2005;34:249-254

DOI: $10.1159 / 000093103$
Received: March 24, 2005

Accepted after revision: July 26, 2005

\title{
A Survey of Thromboprophylaxis Management in Patients with Major Trauma
}

\author{
Davide Imberti $^{a}$ Walter Ageno ${ }^{b}$

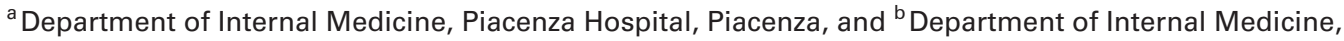 \\ University of Insubria, Varese, Italy
}

\section{Key Words}

Venous thromboembolism - Thromboprophylaxis •

Trauma, major · Heparin, low-molecular-weight •

Prophylaxis, physical · Vena cava filter

\begin{abstract}
Background: Venous thromboembolism (VTE) is a common life-threatening complication of major trauma. Although clinical guidelines clearly suggest routine VTE prevention with low-molecular-weight heparin (LMWH) in this specific group of patients, there is still a lack of strong recommendations regarding the timing and the modality of heparin administration and the role of mechanical prophylaxis. We tested the hypothesis that there is significant practice variation in thromboprophylaxis management of patients with major trauma among intensive care unit (ICU) specialists. Methods: Two hundred Italian ICU specialists, representing 200 ICU throughout the country, were contacted by telephone and were asked (1) whether they routinely prescribe pharmacological antithrombotic prophylaxis in patients with major trauma, whether prophylaxis is prescribed to all patients or to selected patients, and the type of prophylaxis and the timing of administration and (2) whether they recommend physical prevention, whether this is prescribed to all patients or to selected patients, and the type of physical prophylaxis. Results: In patients with major trauma, $85 \%$ of the interviewed ICU specialists an-
\end{abstract}

swered that they prescribe pharmacological prophylaxis for VTE. $37.6 \%$ of them prescribe prophylaxis only to selected patients based on the level of risk, $87.7 \%$ prescribe low-molecular-weight heparin, and $42.4 \%$ start prophylaxis immediately after hospitalization. Only $61 \%$ of the interviewed specialists prescribe physical prophylaxis; $82.8 \%$ of them use elastic stockings, $9.8 \%$ intermittent pneumatic compression, and $7.4 \%$ other mechanical devices. Physical prophylaxis is prescribed to all patients by $41 \%$, and by $59 \%$ only in case of contraindication to pharmacological prevention. Inferior vena cava (IVC) filter insertion is considered by $47 \%$ when anticoagulation is contraindicated; $91.4 \%$ of them recommend the IVC filter only if deep vein thrombosis (DVT) has been diagnosed. Conclusions: Even when there are clinical guidelines, prescription of VTE prevention in patients with major trauma is underused and timing and modality of prophylaxisare ratherheterogeneous. When anticoagulation is contraindicated, IVC filters are commonly recommended only in the presence of DVT.

Copyright (c) 2005 S. Karger AG, Basel

Venous thromboembolism (VTE) is a common, lifethreatening complication of severe trauma. Without prophylaxis, the reported risk of deep vein thrombosis (DVT) in patients with multiorgan or major trauma varies between 10 and 58\% [1-4], with the variation in the rate being attributable to the study design and to the diagnos-

\section{KARGER}

Fax +4161306 1234 E-Mail karger@karger.ch www.karger.com
(C) 2005 S. Karger AG, Basel

$1424-8832 / 05 / 0346-0249 \$ 22.00 / 0$

Accessible online at:

www.karger.com/pht
Dr. Davide Imberti

Thrombosis Center, Internal Medicine Unit

Ospedale Civile, Via Taverna 49

IT-29100 Piacenza (Italy)

Tel. +390523 303 313, Fax +390523 303 315, E-Mail d.imberti@ausl.pc.it 
tic techniques. In a well-conducted prospective study of 443 patients with major trauma [2], the rate of venography-proven DVT was $58 \%$, and $18 \%$ of the patients had proximal DVT. In this clinical setting, the rate of fatal pulmonary embolism (PE) ranges between 0.5 and $2 \%[5$, 6] and PE is the third most common cause of death in patients who survive the first $24 \mathrm{~h}$ [2]. In this population at a very high risk of VTE, the optimal thromboprophylactic management can be very problematic because of the frequently associated increased hemorrhagic risk and because of the inherent heterogeneity of the trauma population. To our knowledge, there are still few trials that have adequately assessed the efficacy and safety of pharmacological and mechanical prophylaxis in patients with major trauma. Several small randomized studies have evaluated unfractioned heparin (UFH) in this setting [4, 7-9], and an important metanalysis [10, 11] clearly demonstrated that UFH is not effective in these patients, with an overall DVT rate of $10 \%$ compared with a $7 \%$ rate in the placebo group. On the other hand, low-molecularweight heparin (LMWH) was shown to be effective in two randomized trials carried out in patients with major trauma. Knudson et al. [12] compared enoxaparin (30 mg twice daily), foot pump and intermittent pneumatic compression (IPC), reporting a DVT rate of 1,7 and $2 \%$, respectively. Haentjens [13] showed in a series of 283 patients with major trauma that a fixed dose of nadroparin (2,850 IU once daily) was at least as effective and safe as a dose of nadroparin adjusted for body weight and time after surgical intervention.

To our knowledge, only one randomized double-blind study using venography as the efficacy end point has compared the efficacy and safety of LMWH and UFH in this clinical setting; in 344 patients with major trauma, a statistically significant reduction in the rate of DVT was observed in the group treated with LMWH with comparable safety results [14].

The use of mechanical prophylactic methods is very attractive in patients with major trauma, especially when heparin prophylaxis is contraindicated. To our knowledge, elastic stockings have never been evaluated in trauma patients, while a number of well-conducted trials have demonstrated an acceptable efficacy of IPC in the prevention of VTE in patients with severe trauma $[4,12,15,16]$. The potential role of the prophylactic inferior vena cava (IVC) filter is still controversial and there are no randomized trials demonstrating a clear benefit of IVC insertion in trauma patients. However, three recent studies have reported a low rate of $\mathrm{PE}$ in patients with severe polytrauma who underwent IVC insertion [17-19]. Currently, IVC filter in- sertion is primarily indicated for patients with demonstrated DVT and contraindications to anticoagulation [20].

Based on the current literature, recommendations for VTE prophylaxis in patients suffering from major trauma are based on the available information derived from the limited number of studies performed in this specific setting in combination with extrapolations from other highrisk groups. In particular, the guidelines of the American College of Chest Physicians (ACCP) [20] strongly recommend the routine use of LMWH in the absence of contraindications due to concomitant bleeding. If $\mathrm{LMWH}$ prophylaxis needs to be delayed or is contraindicated because of concerns about bleeding, mechanical prophylaxis (elastic stockings, IPC) should be used. IVC filters may be inserted if proximal DVT is demonstrated and anticoagulation is contraindicated.

However, there is still little consensus on the timing and on the modality of LMWH administration and on the role of mechanical prophylaxis in case of bleeding contraindications. We tested the hypothesis that there is significant practice variation in thromboprophylaxis management of patients with major trauma among intensive care unit (ICU) specialists.

\section{Methods}

We performed a telephone survey to assess the use of VTE prophylaxis in patients with major trauma among Italian ICU specialists. In Italy, there are 1,212 ICUs. In order to obtain a reliable estimate of clinical practice patterns throughout the country, we identified four regional areas (northwestern, northeastern, central, and southern Italy) and we identified public and private hospitals. Two major variables were considered to calculate the sample size: each interview had to represent a single department of intensive care (i.e. 1 interviewed ICU specialist represented one institution); the characteristics of the sampled departments had to be proportional to the characteristics of all Italian departments regarding the regional distribution, the definition of private or public, and the dimensions of the department based on the number of beds. Using the method described by Diamond and Jefferies [21], the estimated sample size was 200 , with a sampling error of $\pm 4.2 \%$ and a confidence interval of $96 \%$.

The inclusion criteria to select the ICU specialists for the interview were: at least 5 years of working experience following the completion of the residency program and age lower than 60 years. At the beginning of the interview, each participating ICU specialist was asked about the number of times he or she is usually involved in prescribing antithrombotic prophylaxis, about the specialist physician who is usually in charge of prescribing antithrombotic prophylaxis in his/her department, about whether he/she considered there was sufficient evidence in the literature to support the routine use of thromboprophylaxis in this area and about the local use of guidelines on antithrombotic prophylaxis. 


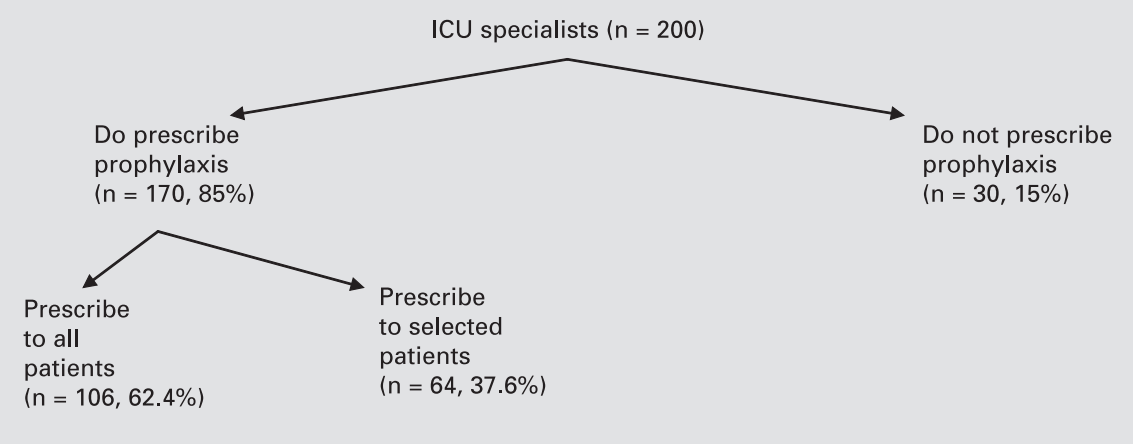

Fig. 1. Prescription of antithrombotic prophylaxis in patients with major trauma.

The first part of the interview was focused on clinical management of pharmacological prophylaxis, the second on mechanical prophylaxis.

In the first part, the interviewed ICU specialists were initially asked about their opinion on whether there is or is not sufficient evidence to prescribe pharmacological antithrombotic prophylaxis (UFH, LMWH or other antithrombotic drugs) to patients with major trauma; whether they prescribe pharmacological antithrombotic prophylaxis in their daily clinical practice to such patients, and if so, whether they prescribe pharmacological antithrombotic prophylaxis to all these patients or just to selected cases. All those who answered 'selected cases' were then asked about which conditions they consider to be relevant for patient risk stratification. These conditions were: age, previous episode of VTE, obesity, varicose veins, concomitant diseases representing a risk of VTE, absence of hemorrhagic contraindications, known thrombophilia, need for surgical procedures, and concomitant use of estrogen therapy. This question allowed multiple answers. At this point, ICU specialists who answered that they do prescribe antithrombotic prophylaxis were asked about which drug they use among low fixed-dose UFH, adjusted-dose UFH, LMWH, or other drugs and what the reason was for preferring it, and finally about the timing when they administer the first dose of the antithrombotic drug.

In the second part, the interviewed ICU specialists were asked whether they prescribe mechanical prophylaxis in their daily clinical practice to patients with major trauma, and if so, whether they prescribe this prophylaxis to all these patients or only in case of hemorrhagic contraindications to pharmacological treatment. At this point, ICU specialists who answered they do prescribe mechanical prophylaxis were asked about which methods they use: elastic stockings, IPC or others. Finally, the interviewed ICU specialists were asked whether they usually consider IVC filter insertion when anticoagulation is contraindicated, and if so, whether they prescribe IVC filters to all these patients or only when DVT has objectively been diagnosed.

The survey was carried out in July 2003. The selection of the sample and the interviews were carried out by a research organization specializing in telephone-based epidemiological surveys (ISIS Research, Milan, Italy). The telephone interviewers were carefully trained regarding the interview technique and the objectives of the research.

\section{Results}

All 200 ICU specialists asked to participate did so. Of them, $95 \%$ are directly involved on a regular basis in the prescription of antithrombotic prophylactic strategies to patients with major trauma; the remainder stated they prescribed antithrombotic prophylaxis less than once a month. In $68 \%$ of the surveyed departments, the ICU specialist is directly responsible for the prescription of antithrombotic prophylaxis. In the remaining $32 \%$ of the cases, the prescribing physician is a surgeon. Eighty-two percent of the interviewed specialists believed there was sufficient evidence in the literature to support the routine prescription of antithrombotic prophylaxis in patients with major trauma. Seventy-two percent of the selected departments adopted or produced internal guidelines for VTE prophylaxis.

The first part of the interview was focused on the clinical management of pharmacological prophylaxis in patients with major trauma. Only $82.5 \%$ of the interviewed ICU specialists believe that there is sufficient evidence to prescribe antithrombotic prophylaxis to this patient population. Pharmacological antithrombotic prophylaxis is routinely prescribed by $85 \%$ of the interviewed ICU specialists. Among the prescribers, $62.4 \%$ prescribe antithrombotic prophylaxis to all patients with major trauma irrespective of the level of risk, whereas $37.6 \%$ select patients on the basis of the concomitant presence of risk factors or in the absence of hemorrhagic contraindications. The rate of prescription of antithrombotic prophylaxis is summarized in figure 1 and risk factors that predict drug selection are summarized in table 1. Of all ICU specialists who prescribe antithrombotic prophylaxis, $87.7 \%$ prescribe LMWH, while $9.4 \%$ prescribe adjusteddose UFH and 2.9\% fixed low-dose UFH. Of interest, the main reason why LMWH is the preferred antithrombot- 
Fig. 2. Prescription of mechanical prophylaxis in patients with major trauma.
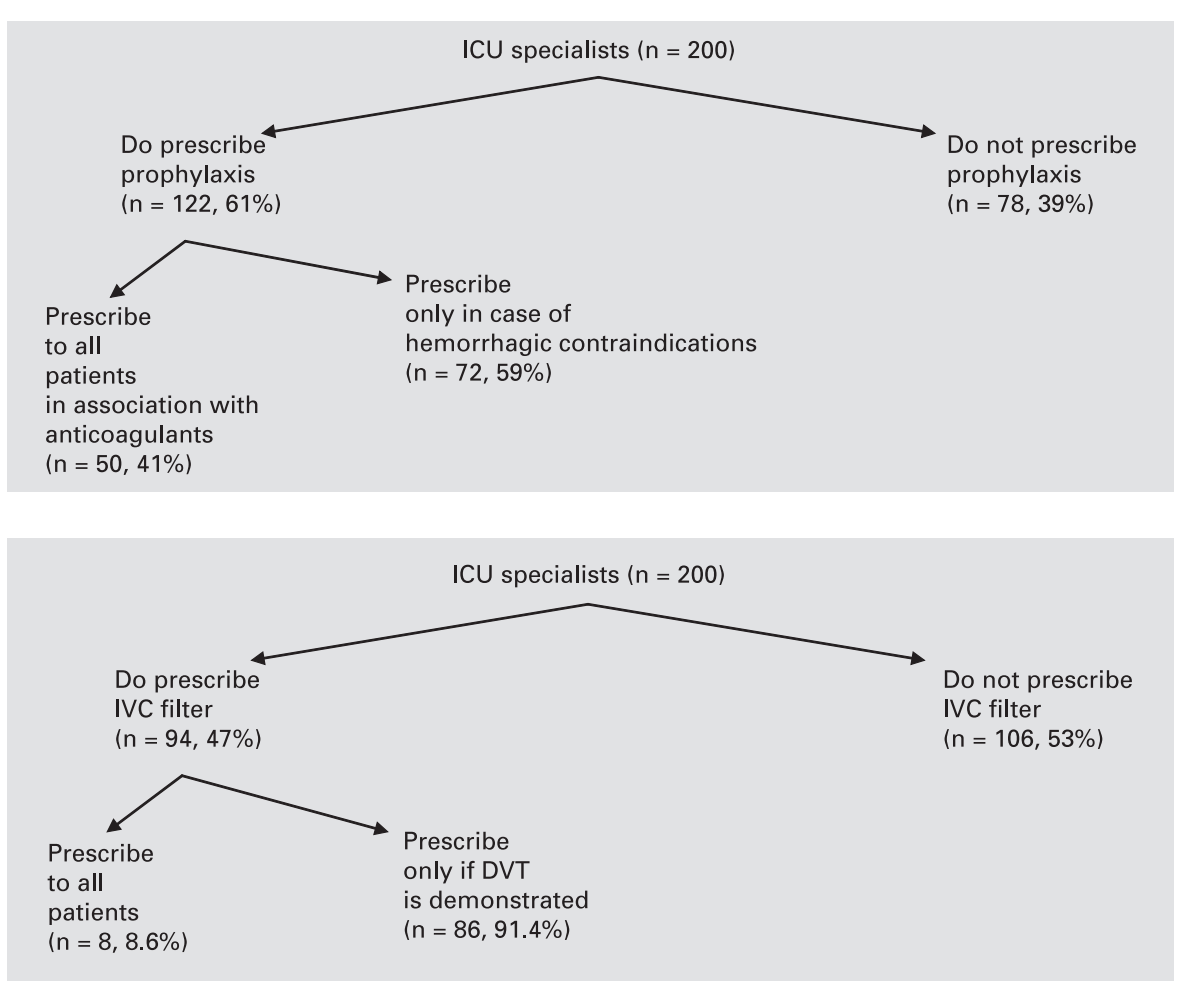

Fig. 3. Prescription of IVC filter in patients with major trauma and hemorrhagic contraindications to pharmacological prophylaxis.
Table 1. Conditions considered to be relevant to decide on the prescription of antithrombotic prophylaxis in patients with major trauma

\begin{tabular}{ll}
\hline & $\%$ \\
\hline Concomitant diseases increasing risk of VTE & 64.1 \\
Age >40 years & 46.9 \\
Previous episode of VTE & 46.9 \\
Varicose veins & 46.9 \\
Obesity & 37.5 \\
Absence of hemorrhagic contraindications & 34.4 \\
Known thrombophilia & 28.1 \\
Surgical intervention associated & 20.3 \\
Estrogen therapy & 17.2 \\
Other & 17.2 \\
\hline
\end{tabular}

a Type of fracture, absence of concomitant head injury, bed rest, associated medical illnesses, platelet count, and aPTT level.

ic drug is the perception that it is safer than UFH. This answer was given by $57.3 \%$ of responders. Only $22 \%$ select LMWH because of a better-perceived efficacy profile. Forty-two percent of the polled physicians initiate prophylaxis immediately after hospitalization, while $35.9 \%$ start prophylaxis in the evening of the first day of hospitalization, 9.8\% do not start immediately, but according to the clinical conditions of the patient, and $5.9 \%$ on the day after hospitalization.

The second part of the interview was focused on the use of mechanical prophylaxis in patients with major trauma. According to the interviewed physicians, only $61 \%$ of the selected ICU specialists prescribe mechanical prophylaxis to patients with major trauma. Among the prescribers, $41 \%$ prescribe physical prophylaxis in association with anticoagulants to all patients with major trauma, whereas $59 \%$ of them do so only in case of the presence of hemorrhagic contraindications to pharmacological prevention. The rate of the prescription of mechanical prophylaxis is summarized in figure 2. Of all ICU specialists who do prescribe mechanical prophylaxis, $82.8 \%$ prescribe elastic stockings, while only $9.8 \%$ prescribe IPC and $7.4 \%$ prescribe other mechanical devices.

In the case of a major contraindication to anticoagulation, only $47 \%$ of the interviewed clinicians would consider IVC filter insertion; among the prescribers, 91.4\% would consider IVC filter insertion only after DVT has been objectively documented. The rate of prescription of IVC filters is summarized in figure 3. 


\section{Discussion}

Despite the presence of clinical guidelines, VTE prophylaxis in patients with major trauma is still quite underused among Italian ICU specialists. Our survey found that $85 \%$ of the interviewed physicians regularly prescribe pharmacological prophylaxis in these patients, and only $62.4 \%$ of them recommend antithrombotic prevention to all patients irrespective of the level of risk, while $37.6 \%$ select patients on the basis of the concomitant presence of risk factors. This finding is quite surprising, because the very high thrombotic risk following major trauma should make VTE prophylaxis mandatory for all patients with major trauma, irrespective of the presence or absence of concomitant risk factors. However, even in very high-risk critically ill patients prophylactic measures are frequently underused [22]. Goldhaber [23] recently reported that in a mixed medical-surgical ICU population DVT prophylaxis was applied only to one third of the patients. Moreover, in a mail survey of 44 ICUs in Canada [24], the directors of the units answered that decisions about the type of VTE prophylaxis employed were made for the most part on a case-by-case basis $(62.1 \%)$, rather than by preprinted orders $(17.2 \%)$, institutional policies $(20.7 \%)$ or practice guidelines $(6.9 \%)$. Several explanations for this underuse have been proposed: physicians may feel that the overall risk of VTE among patients has decreased sufficiently so that prophylaxis is not warranted; clinicians are more concerned about bleeding complications than about thromboembolism, and it is widely believed that the subjective perception of the magnitude of the problem underestimates its true importance [20].

Of interest, in our survey only $87.7 \%$ of the interviewed physicians who prescribe antithrombotic prophylaxis usually prescribe LMWH, while a not irrelevant percentage $(12.3 \%)$ prefer UFH. This habit is in contrast with the data reported in the literature, which clearly show that UFH has little benefit over no prophylaxis in patients with major trauma $[10,11]$ and it is statistically less effective when directly compared to LMWH [14].

Another very important unsolved clinical problem is the optimal timing for starting heparin administration in patients with major trauma, in the light of the very high bleeding risk especially in the first hours after hospital admission. We observed a great heterogeneity in the timing of the starting of prophylaxis, as evidenced by the observation that no strategy was associated with more than 50\% agreement among respondents. This different behavior is likely due to the difficulty to establish the hemostatic stability of the patients based on the clinical as-

Thromboprophylaxis Management in Patients with Major Trauma sessment in a standardized manner, and to the lack of clear guidelines in the literature [20].

The usage rate of mechanical DVT prophylaxis for patients suffering from major trauma is surprisingly very low among Italian ICU specialists, despite the recommendations of the 2004 Consensus Conference of the ACCP [20] and despite the results of an important recent review [5], which strongly suggested physical prevention when anticoagulation is contraindicated. In fact, only $61 \%$ of the interviewed physicians recommend physical prevention; among prescribers, $41 \%$ prescribe physical prophylaxis in association with anticoagulants to all patients with major trauma, whereas $59 \%$ of them only in the case of the presence of hemorrhagic contraindications to pharmacological prevention. Moreover, the most frequently used mechanical devices were elastic stockings (prescribed in $82.8 \%$ of the cases), even though no published randomized trials have ever evaluated their efficacy in trauma patients. Only $9.8 \%$ of the interviewed specialists suggest IPC, which is the most widely validated mechanical device in this clinical setting. Probably, the main reasons for underusing IPC include their inability to be applied in case of lower limb fractures or in case of plaster casts, a poor compliance by patients and nursing staff, the relatively high cost and the insufficient knowledge about their use by physicians.

There was another interesting result of our survey on the use of IVC filters in the presence of bleeding contraindications to pharmacological prophylaxis. Surprisingly, more than half of the interviewed clinicians (53\%) answered that they usually do not consider IVC filter insertion at all even when anticoagulation is contraindicated; otherwise, among prescribers, $91.4 \%$ recommend IVC filter insertion only if DVT has been demonstrated, according to the recommendations of the guidelines of the ACCP [20].

The underuse of these devices may be due mainly to the important limitations of both definitive filters (increased risk of DVT in the long-term follow-up after insertion) and temporary filters (difficult management). An attractive alternative seems to be the use of retrievable filters [25, 26], which is particularly appealing for patients with major trauma, in whom the risk of PE remains high for a relatively short period of time. In a recent survey, 620 trauma surgeons revealed that the potential removability of these retrievable IVC filters would significantly increase the prophylactic placement rate from 29 to $53 \%$ [27].

Our results are limited in part by the design of the study. The opinion of a single physician may not ade- 
quately reflect the clinical practice of the entire department where he/she works and may also not accurately reflect true prescribing habits [20]. Moreover, the answer to a telephone interview may not entirely reflect the real prescription habit of the interviewed specialist.

In conclusion, even in the presence of clinical guidelines, both pharmacological and mechanical antithrombotic prevention in patients with major trauma is still underused. Timing and modality of prophylaxis are rather heterogeneous. If pharmacological prophylaxis is contraindicated, the possibility of IVC filter insertion is taken into consideration only in $50 \%$ of the cases.
The interesting results of this survey strongly suggest that additional high-quality investigations on antithrombotic prophylaxis are required in this setting.

\section{Acknowledgments}

We are indebted to Dr. Maria Pia Bertuzzi for her help in the linguistic revision of the paper.

This study was supported by an educational grant from Astra Zeneca (Milan, Italy).

\section{References}

$\checkmark 1$ Kudsk KA, Fabian TC, Baum S, et al: Silent deep vein thrombosis in immobilized multiple trauma patients. Am J Surg 1989;158:515519.

-2 Geerts WH, Code JI, Jay RM, et al: A prospective study of venous thromboembolism after major trauma. N Engl J Med 1994;331:16011606.

$>3$ Burns GA, Cohn SM, Frumento RJ, et al: Prospective ultrasound evaluation of venous thrombosis in high-risk trauma patients. J Trauma 1993;35:405-408.

4 Knudson MM, Lewis FR, Clinton A, et al: Prevention of VTE in trauma patients. J Trauma 1994;37:480-487.

$\checkmark 5$ Rogers FB: Venous thromboembolism in trauma patients: a review. Surgery 2001;130:112.

6 Rocha AT, Tapson VF: Venous thromboembolism in intensive care patients. Clin Chest Med 2003;24:103-122.

7 Pasquale M, Fabian TC: Practice management guidelines for trauma from the Eastern Association for the Surgery for Trauma. J Trauma 1998;44:941-957.

8 Shackford SR, Davis JW, Hollingworth-Fridlund $\mathrm{P}$, et al: Venous thromboembolism in patients with major trauma. Am J Surg 1990;159: 365-369.

$\checkmark 9$ Napolitano LM, Garlapati VS, Heard SO, et al: Asymptomatic deep vein thrombosis in the trauma patients: is an aggressive screening protocol justified? J Trauma 1995;39:651-659.

10 Upchurch GR, Demling RH, Davies J, et al: Efficacy of subcutaneous heparin in prevention of venous thromboembolic events in trauma patients. Am Surg 1995;61:749-755.
1 Rogers FB, Cipolle MD, Velmahos G, et al: Practice management guidelines for the prevention of venous thromboembolism in trauma patients: the EAST practice management guidelines work group. J Trauma 2002;53 142-164.

12 Knudson MM, Morabito D, Paiement GD, et al: Use of low molecular weight heparin in preventing thromboembolism in trauma patients. J Trauma 1996;41:446-459.

13 Haentjens P: Thromboembolic prophylaxis in orthopedic trauma patients: a comparison between a fixed dose and an individually adjusted dose of low molecular weight heparin. Injury 1996;27:385-390.

14 Geerts WH, Jay RM, Code KL, et al: A comparison of low dose heparin with low molecular weight heparin as prophylaxis against venous thromboembolism after major trauma. N Engl J Med 1996;335:701-707.

15 Elliott CG, Dudney TM, Egger M, et al: Calfthigh sequential pneumatic compression compared with plantar venous pneumatic compression to prevent deep-vein thrombosis after non-lower extremity trauma. J Trauma 1999; 47:25-32.

16 Satiani B, Falcone R, Shook L, et al: Screening for major deep vein thrombosis in seriously injured patients: a prospective study. Ann Vasc Surg 1997;11:628-629.

17 Gosin IS, Graham AM, Ciocca RG, et al: Efficacy of prophylactic vena cava filters in high risk trauma patients. Ann Vasc Surg 1997;11: 100-105.
18 Rogers FB, Shackford SR, Ricci MA, et al: Routine prophylactic vena cava filter insertion in severely injured patients decreases the incidence of pulmonary embolism. J Am Coll Surg 1995; 180:641-647.

19 Spain DA, Richardson JD, Polk HC, et al: Venous thromboembolism in the high-risk trauma patient: do risks justify aggressive screening and prophylaxis? J Trauma 1997;42:463467.

20 Geerts WH, Pineo GF, Heit JA, et al: Prevention of venous thromboembolism. Chest 2004; 126(suppl 3):338S-380S.

21 Diamond I, Jefferies J: Statistics for Social Sciences. London, McGraw Hill, 2001.

22 Keane MG, Ingenito EP, Goldhaber SZ: Utilization of venous thromboembolism prophylaxis in the medical intensive care units. Chest 1994;106:13-14

23 Goldhaber SZ: Venous thromboembolism in the intensive care unit: the last frontier for prophylaxis. Chest 1998;113:5-7.

24 Cook D, McMullin J, Hodder R, et al: Prevention and diagnosis of venous thromboembolism in critically ill patients: a Canadian survey. Crit Care 2001;5:336-342.

25 Neuerburg JM, Gunther RW, Vorwerk D, et al: Results of a multicenter study of the retrievable tulip vena cava filter: early clinical experience. Cardiovasc Intervent Radiol 1997;20 $10-16$.

26 Bovyn G, Gory P, Reynaud P, et al: The tempofilter: a multicenter study of a new temporary caval filter implantable for up to six weeks. Ann Vasc Surg 1997;11:520-528.

-27 Quirke TE, Ritota PC, Swan KG: Inferior vena cava filter use in the US trauma centers: a practitioner's survey. J Trauma 1997;43:333-337. 